\title{
Diagnostic values of serum tumor markers CA72-4, SCCAg, CYFRA21-1, NSE, AFU, CA125, CA19-9, CEA and FER in nasopharyngeal carcinoma
}

\author{
Yong-Fei Tang ${ }^{1}$, Wen $\mathrm{Li}^{2}$, Jing-Ping Yuan ${ }^{1}$, Ya-Bing Huang ${ }^{1}$, Hong-Lin $\mathrm{Yan}^{1}$, Lin Liu ${ }^{1}$, Xiang-Pan Li ${ }^{3}$, \\ Ming Wang ${ }^{4}$ \\ ${ }^{1}$ Department of Pathology, ${ }^{2}$ Department of Hematology, ${ }^{3}$ Department of Oncology, ${ }^{4}$ Department of Clinical Laboratory, Renmin Hospital of Wuhan \\ University, Wuhan 430060, China \\ Contributions: (I) Conception and design: M Wang, YF Tang; (II) Administrative support: JP Yuan; (III) Provision of study materials or patients: \\ M Wang, YF Tang; (IV) Collection and assembly of data: W Li, YF Tang, YB Huang, XP Li; (V) Data analysis and interpretation: W Li, HL Yan, \\ L Liu; (VI) Manuscript writing: All authors; (VII) Final approval of manuscript: All authors. \\ Correspondence to: Ming Wang. Department of Clinical Laboratory, Renmin Hospital of Wuhan University, Wuhan 430060, China. \\ Email: morgan@whu.edu.cn.
}

Background: The purpose of this study was to explore the clinical diagnostic values of serum tumor markers (TMs) including squamous cell carcinoma antigen (SCCAg), cytokeratin 19 fragment (CYFRA21-1), neuron specific enolase (NSE), alpha-L-fucosidase (AFU), carbohydrate antigen (CA)72-4, CA125, CA19-9, carcinoembryonic antigen (CEA) and ferritin (FER) in nasopharyngeal carcinoma (NPC).

Methods: Two hundred and two newly diagnosed NPC patients and seventy-two benign nasopharyngeal diseases (BND) patients were retrospective reviewed. The levels of serum CA72-4, CYFRA21-1, SCCAg, NSE, AFU, FER, CEA, CA125 and CA19-9 were measured by chemiluminescent assay. AFU serum levels were detected by using continuous monitoring method. The non-parametric Mann-Whitney $\mathrm{U}$ test was used to compare median levels of TMs between the benign group and NPC group. Logistic stepwise regression analysis and the receiver operating characteristic (ROC) curve were firstly used in screening TMs in NPC, and then the decision tree was applied to evaluate the diagnostic value of TMs in NPC.

Results: The levels of serum CA72-4, CYFRA21-1, NSE and FER in patients with NPC were significantly higher than those with the BND $(\mathrm{P}<0.05)$. For CEA, SCCAg, AFU, CA19-9 and CA125, there is no significant difference in the serum of NPC and benign control $(\mathrm{P}>0.05)$. AFU and CA72-4 were eliminated by Logistic regression stepwise analysis. Logistic regression showed that CA72-4, CYFRA21-1, NSE, CA125, CA19-9, CEA and FER OR (95\% CI) of risk of NPC events were 1.09 (0.96-1.24, P=0.176), 2.42 (1.66-3.68, $\mathrm{P}<0.001), 1.30$ (1.16-1.46, $\mathrm{P}<0.001), 0.96$ (0.92-1, $\mathrm{P}=0.043), 1.042$ (0.994-1.0994, $\mathrm{P}=0.099)$, $0.67(0.47-0.94, \mathrm{P}=0.022)$ and $1.003(1.0007-1.005, \mathrm{P}=0.014)$. NSE and CYFRA21-1 were selected for the conditional inference tree (CTree). When NSE $>15.90$, the positive predictive value was $93.9 \%$. In the lower NSE group, when the CYFRA21-1 was greater than 3.3, the positive predictive value was $92.3 \%$.

Conclusions: The combination of CYFRA21-1 and NSE will be beneficial for distinguishing NPC from BND.

Keywords: CYFRA21-1; neuron specific enolase (NSE); nasopharyngeal carcinoma; diagnosis; the decision tree

Submitted Apr 27, 2018. Accepted for publication Oct 24, 2018.

doi: $10.21037 /$ tcr.2018.10.24

View this article at: http://dx.doi.org/10.21037/tcr.2018.10.24

\section{Introduction}

Nasopharyngeal carcinoma (NPC) is a disease in which malignant (cancer) cells are found in the tissues of the nasopharyngeal. It is one of the most prevalent malignancies in southern China, Southeast Asia, the Arctic, and the Middle East/North Africa $(1,2)$. Atypical symptoms and 
hidden sites in earlier stage of nasopharyngeal carcinoma are the main causes of a high misdiagnosis rate. Thus, it has been diagnosed already in middle or late period (3). With the wide application of tumor markers (TMs) in nasopharyngeal carcinoma, exploring a highly sensitive and specific biomarker for early diagnosis of NPC is extremely important to improve the survival rate.

There are many TMs such as squamous cell carcinoma antigen (SCCAg), cytokeratin 19 fragment (CYFRA21-1), neuron specific enolase (NSE), alpha-L-fucosidase (AFU), carbohydrate antigen (CA)72-4, CA125, CA19-9, carcinoembryonic antigen (CEA) and ferritin (FER) were the most commonly used in the clinic. Besides, CYFRA was also reported to be important significance in the differential diagnosis for the nasopharyngeal carcinoma and benign disease $(4,5)$, and was used as a better TM than SCC antigen for detection of NPC (6). While, whether the other TMs can also be used for diagnosis of NPC, there is still unknown. Logistic regression analysis and the decision tree were used to screen TMs. Decision tree is a novel data processing method, which has been applied to epidemiological studies $(7,8)$.

The objective of this study was to analyze the potential diagnostic value of serum TMs for distinguishing of early-stage NPC from various benign nasopharyngeal diseases (BND).

\section{Methods}

\section{Patients}

This study was approved by the institutional review board (IRB) of hospital, and a signed consent form was obtained from each patient. Between January 2012 and October 2017, 202 newly diagnosed NPC patients and 72 BND patients were finally enrolled by excluding missing data. All patients satisfied the inclusion criteria: (I) clinical and pathological data were completely recorded. (II) All cases were proved by pathology and classified according to World Health Organization (WHO) 2005 classifications and AJCC 7th edition guidelines of TNM classification, respectively $(9,10)$. (III) There is no other malignant tumor. (IV) Postoperative pathologic type of nasopharyngeal carcinoma: keratinizing squamous cell carcinoma (type I); and nonkeratinizing carcinoma, characterized as differentiated (type II) or undifferentiated (type III). BND, composed of nasal polyps and nasopharyngeal chronic inflammatory. (V) TMs were measured within 24 h-3 $\mathrm{d}$ before radiotherapy and chemotherapy. All patients were first diagnosed without any treatment before.

\section{Detection of TMs}

The turnaround time from specimens collecting from patients to assay detection was within 2 hours. The samples were centrifuged for 15 minutes at 3,000 g. CA72-4, CYFRA21-1, NSE, CA125, CA19-9, CEA and FER levels were measured by a cobas e601 analyzer (Roche, USA). The serum levels of SCCAg was detected by an ARCHITECT i2000 immunoassay analyzer (Abbott, USA). AFU was measured by an automatic biochemistry analyzer ADVIA 2400 analyzer (Siemens, USA).

\section{Statistical analysis}

The normal distribution test was conducted in the variables by Shapiro-Wilk method. As the TM levels were not normally distributed, the results of the TM level were reported as median. The differences in serum levels of TMs were analyzed using non-parametric Mann-Whitney $\mathrm{U}$ test. The enumeration data were performed with the chi-square test. The sensitivity, specificity and diagnostic cutoff levels were calculated by receiver operating characteristic (ROC) curve. Levels of TMs were analyzed by logistic stepwise regression analysis (MASS packages) and the decision tree CTree (party packages). All statistical analyses were performed using $\mathrm{R}$ (version 3.3.2), $\mathrm{P}<0.05$ was considered to be a statistically significant.

\section{Results}

\section{Patient characteristics}

The characteristics of 202 NPC patients and 72 patients with BND are shown in Table 1. The male to female ratio was 153:49 (3.12:1) in patients with NPC, and another group is 50:22 (2.27:1). Median age in NPC patients was 51 (range, $16-83)$ years. The median age of patients with BND was 50 (range, 14-88) years. NPC samples included 179 cases of non-squamous cell carcinoma with undifferentiation and 25 cases of non-squamous cell carcinoma with differentiation. BND samples included nasal polyps $(n=38)$ and nasopharyngeal chronic inflammatory $(n=34)$. There was no difference on two groups by age, gender $(\mathrm{P}>0.05)$.

\section{The levels of TMs in all patients}

The levels of serum CA72-4, CYFRA21-1, SCCAg, NSE, AFU, CA125, CA19-9, CEA and FER in all patients are shown in Table 2. The levels of serum CA72-4, CYFRA21-1, 
Table 1 Characteristics of study population

\begin{tabular}{|c|c|c|}
\hline Clinical characteristics & NPC $(n=202)$ & BND $(n=72)$ \\
\hline \multicolumn{3}{|l|}{ Gender } \\
\hline Male & 153 & 50 \\
\hline Female & 49 & 22 \\
\hline \multicolumn{3}{|l|}{ Age, years } \\
\hline Median & 51 & 50 \\
\hline Range & $16-83$ & $14-88$ \\
\hline \multicolumn{3}{|l|}{ Stage } \\
\hline I & 12 & - \\
\hline II & 44 & - \\
\hline III & 84 & - \\
\hline IV & 62 & - \\
\hline \multicolumn{3}{|l|}{ Histology } \\
\hline Type I & 0 & - \\
\hline Type II & 25 & - \\
\hline Type III & 177 & - \\
\hline \multicolumn{3}{|c|}{ Benign nasopharyngeal diseases } \\
\hline Nasal polyps & - & 38 \\
\hline $\begin{array}{l}\text { Nasopharyngeal chronic } \\
\text { inflammatory }\end{array}$ & - & 34 \\
\hline
\end{tabular}

NSE and FER in patients with NPC and early stage NPC (stages I and II) were separately significantly higher than those of BND $(\mathrm{P}<0.05)$. No significant difference in the levels of serum SCCAg, AFU, CA125, CA19-9 and CEA was observed among patients with NPC or early stage NPC and $\mathrm{BND}(\mathrm{P}>0.05)$.

The levels of TMs in gender, histology and TNM stage are shown in Table 3. The levels of serum SCCAg, CYFRA21-1, CEA and FER in male were significantly higher than in female $(\mathrm{P}<0.05)$. No different $T M$ s were observed between differentiated and undifferentiated nonkeratinizing carcinoma ( $>0.05)$. Only CYFRA21-1 has the remarkable difference in early and middle-late stage $(\mathrm{P}<0.05)$.

\section{Logistic regression analysis}

AFU and SCC were eliminated by Logistic regression stepwise analysis. The residual TMs were refitted and the regression equation model: $\mathrm{Y}=\operatorname{logit}(\mathrm{P})=-5.288+0.839 \mathrm{X}_{\mathrm{C}}$ $\mathrm{YFRA}+0.240 \mathrm{X}_{\mathrm{NSE}}+0.003 \mathrm{X}_{\mathrm{FER}}$. Logistic regression showed that CYFRA21-1, NSE and FER OR (95\% CI) of risk of NPC events were 2.315 (1.617-3.314, $\mathrm{P}<0.001), 1.272$ $(1.147-1.410, \mathrm{P}<0.001)$ and $1.003(1.001-1.005, \mathrm{P}=0.014)$.

The ROC of serum Y, CYFRA21-1, NSE and FER in NPC patients was shown in Figure 1. The area under curve (AUC), cutoff levels, sensitivities and specificities of serum TMs in patients with NPC were shown in Table 4.

Table 2 The levels of tumor markers in patients with NPC and BND

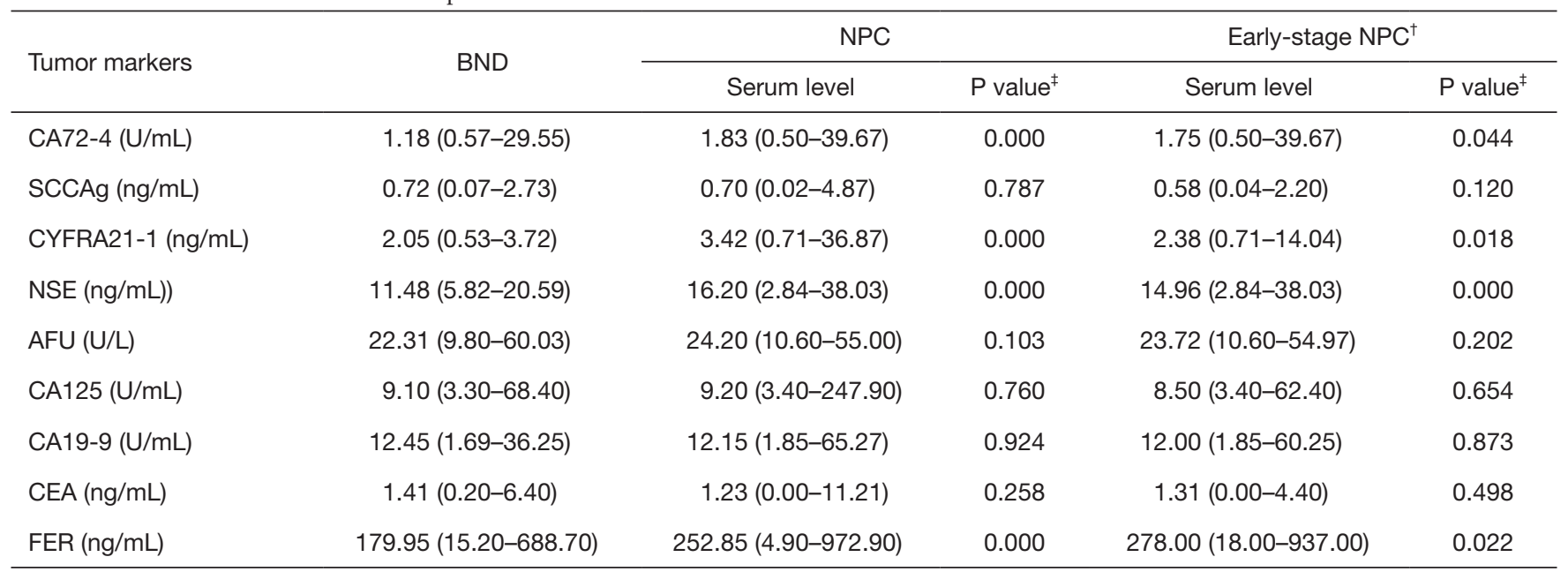

${ }^{\dagger}$, stages I and II; ${ }^{\ddagger}$, compared with BND. NPC, nasopharyngeal carcinoma; BND, benign nasopharyngeal diseases. 
Table 3 The levels of tumor markers in histology, TNM stage and gender

\begin{tabular}{|c|c|c|c|c|c|c|c|c|c|}
\hline Tumor markers & \multicolumn{3}{|c|}{ Histology } & \multicolumn{3}{|c|}{ TNM stage } & \multicolumn{3}{|c|}{ Gender } \\
\hline CA72-4 (U/mL) & 1.57 & 1.90 & 0.477 & 1.82 & 2.11 & 0.228 & 1.83 & 1.90 & 0.718 \\
\hline $\operatorname{sCCAg}(n g / m L)$ & 0.71 & 0.70 & 0.594 & 0.57 & 0.71 & 0.061 & 0.70 & 0.59 & 0.033 \\
\hline CYFRA21-1 (ng/mL) & 2.95 & 3.48 & 0.165 & 2.50 & 3.76 & 0.000 & 3.42 & 3.15 & 0.012 \\
\hline AFU (U/L) & 23.23 & 24.99 & 0.454 & 24.41 & 23.19 & 0.990 & 24.20 & 23.45 & 0.922 \\
\hline CA125 (U/mL) & 9.40 & 9.20 & 0.708 & 8.50 & 9.30 & 0.138 & 9.20 & 9.20 & 0.511 \\
\hline CA19-9 (U/mL) & 13.01 & 12.11 & 0.904 & 12.00 & 12.61 & 0.829 & 12.16 & 12.48 & 0.197 \\
\hline CEA (ng/mL) & 0.85 & 1.28 & 0.094 & 1.35 & 1.22 & 0.553 & 1.23 & 0.95 & 0.023 \\
\hline
\end{tabular}

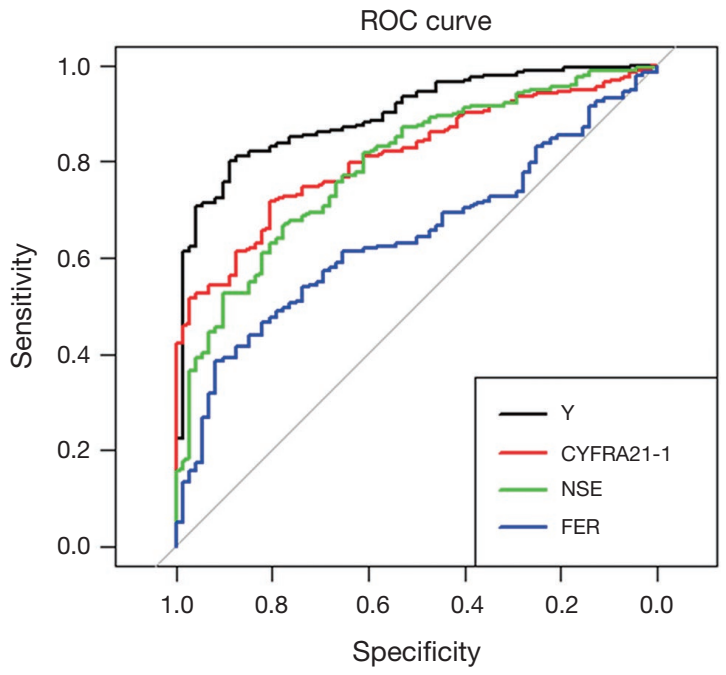

Figure 1 ROC curve of three serum tumor markers in NPC patients. The ROC curve showing significantly higher specificity of FER as compared to CYFRA21-1 and NSE and significantly higher sensitivity of CYFRA21-1 as compared to and NSE and FER. ROC, receiver operating characteristic; NPC, nasopharyngeal carcinoma.

The results indicated that $\mathrm{Y}$ and FER had the specificities of $90.3 \%$ and $91.7 \%$ for NPC, significantly higher than that of CYFRA21-1, NSE ( $\mathrm{P}<0.05)$. Y had the sensitivity of $74.8 \%$ for NPC, significantly higher than that of CYFRA21-1, NSE and FER $(\mathrm{P}<0.05)$. Regression model $\mathrm{Y}$ had the largest AUC area and the greatest sensitivity and specificity.

\section{The decision tree}

The conditional inference tree (CTree) showed that only NSE and CYFRA21-1 were selected as the differential diagnosis of NPC and BND (Figure 2). The CTree algorithm produced a tree with 7 terminals. Splitting the population according to the serum level of NSE $\leq 11.53$ versus $>11.53$ produces two subgroups. The lower NSE group (NSE $\leq 11.53)$ again splits into two groups: CYFRA2 $1-1 \leq 3.3$ and CYFRA21-1 $>3.3$. In the higher NSE group (NSE $>11.53$ ), further splits into subgroups according to NSE $\leq 15.91$ and NSE $>15.91$. These nodes defined four distinct subgroups: when NSE $\leq 11.53$ and CYFRA21-1 $\leq 3.3$, the negative predictive value was $72.5 \%$. When NSE was between 11.53 and 15.91 , the positive predictive value was $72.2 \%$. When NSE $\leq 11.53$ and CYFRA21-1 $>3.3$, the positive predictive value was $92.3 \%$. When NSE $>15.91$, its positive predictive value was $93.8 \%$.

\section{Discussion}

In this study, nine common TMs were detected to analyze the diagnostic value of nasopharyngeal carcinoma. We found that the levels of serum SCCAg, AFU, CA125, CA19-9 and CEA showed no significant difference between patients with NPC and BND, which means that SCCAg, AFU, CA125, CA199 and CEA had no diagnostic value for patients with NPC. The levels of serum CA72-4, CYFRA21-1, NSE and FER were significantly increased in patients with NPC, indicating that they have a certain diagnostic value in patients with NPC. The different levels of CYFRA21-1 and FER between 
Table 4 The area under curve (AUC), cutoff levels, sensitivities, specificities, and accuracies of serum TMs

\begin{tabular}{lccccc}
\hline Tumor marker & AUC & Cutoff & Specificity (\%) & Sensitivity (\%) & Accuracy (\%) \\
\hline Y & 0.881 & - & 90.3 & 74.8 & 81.8 \\
CYFRA21-1 & 0.810 & 2.565 & 80.6 & 71.8 & 74.8 \\
NSE & 0.790 & 14.26 & 77.8 & 66.8 & 74.5 \\
FER & 0.641 & 318.65 & 91.7 & 38.6 & 74.1 \\
\hline
\end{tabular}

NSE, neuron specific enolase; FER, ferritin; TMs, tumor markers.

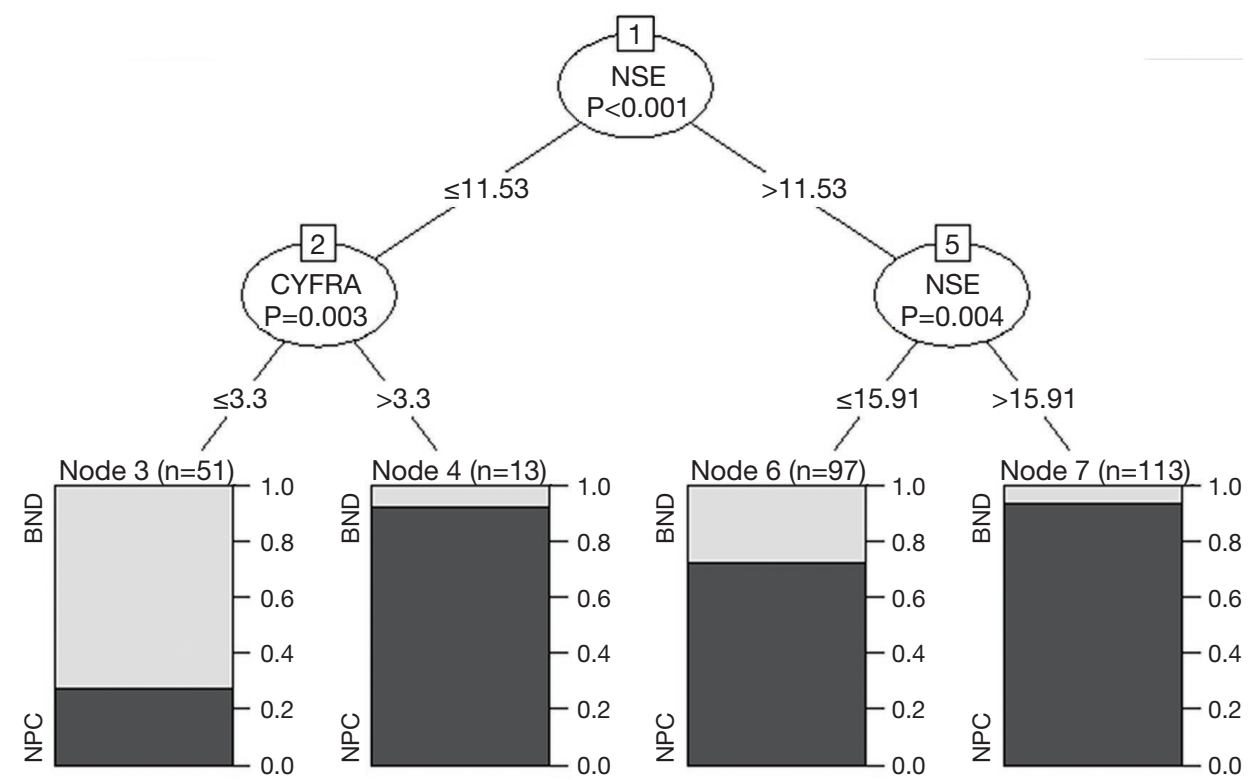

Figure 2 Decision tree model for predicting NPC. The number of patients (n) are given in terminal nodes. The black shadow represents the percentage of NPC in this group. NPC, nasopharyngeal carcinoma; NSE, neuron specific enolase; BND, benign nasopharyngeal diseases.

in male and female was derived from a higher incidence and mortality of NPC in male (2).

Logistic regression analysis and ROC curve was conducted to evaluate the value of serum TMs in the diagnosis of NPC. The study showed that CYFRA21-1, NSE, and FER were considered to be the predictors of NPC, of which CYFRA21-1 was the strongest predictor $(\mathrm{OR}=2.315)$. CA72-4 was not significantly associated with the risk of NPC events, therefore, it was not considered to be the predictor of nasopharyngeal carcinoma.

In this study, the research results showed that the specificities of FER and $\mathrm{Y}$ were $91.7 \%$ and $90.3 \%$ respectively, significantly higher than the specificities of CYFRA21-1 and NSE. The sensitivities of $Y$ significantly higher than CYFRA21-1, NSE and FER. This suggests that the specificity and sensitivity of the regression model were higher than all the single TMs described in the article. Of course, the regression model also had disadvantages, which was more complicated.

The main decision tree methods in the $\mathrm{R}$ statistical were CART and CTree. CTree technology had advantages that were simpler operation and easier to interpretation of the results than CART. Therefore, the CTree method was used in this study (11). The results of the decision tree form a flow chart which is closer to the clinical practice. Only NSE and CYFRA21-1 were considered to be associated with the differential diagnosis of NPC. NSE appears twice as a split node, which results in terminal nodes 6 and 7, and was a very important serum tumor marker. When NSE $>15.91$, the positive predictive value was $93.8 \%$. In the lower NSE group, when the CYFRA21-1 was greater than 3.3, the positive predictive value was $92.3 \%$. All two groups were given a good positive predictive value. The positive predictive value 
between 11.53 and 15.91 was $72.2 \%$, additional detection methods were needed to distinguish them. The decision tree model was poor for negative prediction because $27.5 \%$ of the nasopharyngeal cancer patients were considered negative.

Serum CYFRA21-1 was 19 soluble fragments of keratin that was found in epithelial cells of a malignant tumor (12). The CYFRA21-1 was associated with the prognosis, metastasis and differentiation of nasopharyngeal carcinoma. Ma et al. demonstrated that serum CYFRA21-1 levels which taken before radiotherapy and chemotherapy predicted survival in patients with nonmetastatic (13). The serum level of CYFRA21-1 without radiation and chemotherapy would be a reliable biomarker to evaluate the long-term prognosis of patients with NPC (14). Song et al. indicated that the AUC, sensitivity and specificity of elevated serum CYFRA21-1 in patients with NPC were $0.91,83 \%$ and $89 \%$, respectively (4). In this study, CYFRA21-1 was not only the strongest predictor $(\mathrm{OR}=2.315)$, but also an important split node in the decision tree. At the same time, it increased along with the progress of the disease. The sensitivity and specificity of CYFRA21-1 were 71.8\% and $80.6 \%$, and it was slightly below compared with that reported in the literature. Different control group may be partly responsible for the difference in the study results.

NSE was known as a serum marker of small cell lung carcinoma (SCLC), retinoblastoma and neuroblastoma $(15,16)$. In this article, the sensitivity and specificity of NSE were $66.8 \%$ and $77.8 \%$. NSE was also an important predictor (OR =1.272), second only to CYFRA21-1. NSE was also a vital node that formed the decision tree with CYFRA21-1. However, the level of serum NSE showed no significant difference between patients with early and middle-late stage groups NPC. NSE was not a useful marker for NPC aggressiveness, but a predictive marker.

SCCAg was a subunit of squamous epithelial cell associated antigen TA-4 and one member of serine protease inhibition of glycoprotein family (17). No difference was observed for SCCAg not only in NPC and benign disease groups, but also in the early and middle-late stage NPC. Kimura and Barak have demonstrated that the level of serum SCCAg was increased in head and neck squamous cell carcinoma $(13,18)$, which was inconsistent with our results. The reason we speculated here is that the research object of these documents are the head and neck cancer, of which only a small amount of NPC. Kearsley states that cellular SCCAg expression was related to the level of cellular differentiation in squamous epithelia. As the worse the differentiation, the weaker the expression. SCCAg expression was consistently absent in poorly differentiated (11). It suggested that the main reason for the low expression of SCCAg may be that the cases of NPC we have studied were undifferentiated squamous cell carcinoma, although there were differences in paraffin samples and serum samples.

Elevated FER levels have been detected in a variety of malignancies $(19,20)$. Similarly, we found that FER was also higher in NPC, but did not increased along with the progress of the disease. FER had little effect on NPC prediction (OR $=1.003)$, meanwhile, it was also eliminated by the decision tree. Serum FER levels were revealed to be not associated with age, survival and recurrence rates on multivariate analysis (12).

These nine TMs were analyzed to determine their significance for the diagnosis of NPC. Firstly, we used nonparametric Mann-Whitney $\mathrm{U}$ test to exclude SCCAg, AFU, CA125, CA19-9 and CEA that's CA72-4, CYFRA21-1, NSE and FER left. And then we further ruled out CA72-4 by Logistic regression analysis, leaving CYFRA21-1, NSE and FER. And the corresponding the value of $\mathrm{OR}$ were obtained, we found that CYFRA21-1 was the strongest predictor, followed by NSE, and FER the weakest. Finally, the decision tree was used to exclude FER, and the remaining NSE and CYFRA21-1. NSE and CYFRA21-1 formed the tree structure of the twolayer branches, similar to the flow chart. CYFRA21-1 and NSE combination of serum TMs can improve diagnostic sensitivity and specificity, and facilitates the early diagnosis and differential diagnosis. Of course, this research had some limitations, which was a retrospective single center analysis.

In conclusion, the combination of CYFRA21-1 and NSE will be beneficial for distinguishing NPC from BND. Decision tree model may help clinicians to conveniently predict NPC, and provide guidance in choosing suitable detection and treatment. CA72-4, SCCAg, AFU, CA125, CA19-9, CEA and FER did not have diagnostic value of the NPC.

\section{Acknowledgments}

Funding: This work was supported by the Hubei Chenguang Talented Youth Development Foundation (No. 2015B29) and the Applied Basic Research Program of Wuhan Science and Technology Bureau (No. 2015060101010048).

\section{Footnote}

Conflicts of Interest: All authors have completed the ICMJE uniform disclosure form (available at http://dx.doi. org/10.21037/tcr.2018.10.24). The authors have no conflicts of interest to declare. 
Ethical Statement: The authors are accountable for all aspects of the work in ensuring that questions related to the accuracy or integrity of any part of the work are appropriately investigated and resolved. The study was conducted in accordance with the Declaration of Helsinki (as revised in 2013). The institutional research ethics committee approved the experimental protocols, the number of ethics approval was 20140036. Informed consent was obtained from all patients.

Open Access Statement: This is an Open Access article distributed in accordance with the Creative Commons Attribution-NonCommercial-NoDerivs 4.0 International License (CC BY-NC-ND 4.0), which permits the noncommercial replication and distribution of the article with the strict proviso that no changes or edits are made and the original work is properly cited (including links to both the formal publication through the relevant DOI and the license). See: https://creativecommons.org/licenses/by-nc-nd/4.0/.

\section{References}

1. Chang ET, Adami HO. The enigmatic epidemiology of nasopharyngeal carcinoma. Cancer Epidemiol Biomarkers Prev 2006;15:1765-77.

2. Wei KR, Zheng RS, Zhang SW, et al. Nasopharyngeal carcinoma incidence and mortality in China in 2010. Chin J Cancer 2014;33:381-7.

3. Janvilisri T. Omics-based identification of biomarkers for nasopharyngeal carcinoma. Dis Markers 2015;2015:762128.

4. Song XM, Wang SZ, Wang ZJ, et al. Serum CYFRA21-1 as an effective tumor biomarker for patients with nasopharyngeal carcinoma. Neoplasma 2015;62:124-9.

5. Song XM, Wang ZJ, Cao WJ, et al. The value of circulating CYFRA21-1 expression in patients with nasopharyngeal carcinoma: a study of 529 subjects. Int J Clin Oncol 2016;21:1038-45.

6. Lee JK, Hsieh JF, Tsai SC, et al. Comparison of CYFRA 21-1 and squamous cell carcinoma antigen in detecting nasopharyngeal carcinoma. Ann Otol Rhinol Laryngol 2001;110:775-8.

7. Aguiar FS, Almeida LL, Ruffino-Netto A, et al. Classification and regression tree (CART) model to predict pulmonary tuberculosis in hospitalized patients. BMC Pulm Med 2012;12:40.

8. Gass K, Klein M, Chang HH, et al. Classification and regression trees for epidemiologic research: an air pollution example. Environ Health 2014;13:17.

9. Edge SB, Compton CC. The American Joint Committee on
Cancer: the 7th edition of the AJCC cancer staging manual and the future of TNM. Ann Surg Oncol 2010;17:1471-4.

10. Barnes L, Eveson JW, Reichart P, et al. World Health Organization classification of tumours: pathology and genetics of head and neck tumours. 3rd ed. Lyon: IRAC Press, 2005.

11. Kearsley JH, Stenzel DJ, Sculley TB, et al. Cellular localisation of tumour antigen (TA-4) in normal, dysplastic and neoplastic squamous epithelia of the upper aerodigestive tract. Br J Cancer 1990;61:631-5.

12. Moll R, Divo M, Langbein L. The human keratins: biology and pathology. Histochem Cell Biol 2008;129:705-33.

13. Ma BB, Leungm SF, Hui EP, et al. Prospective validation of serum CYFRA 21-1, beta-2-microglobulin, and ferritin levels as prognostic markers in patients with nonmetastatic nasopharyngeal carcinoma undergoing radiotherapy. Cancer 2004;101:776-81.

14. Wei Z, Zeng X, Xu J, et al. Prognostic value of the pretreatment serum level of cytokeratin fraction 21-1 in undifferentiated nasopharyngeal carcinoma: a study of 332 cases. Head Neck 2014;36:71-6.

15. Kuralay F, Tokgoz Z, Comlekci A. Diagnostic usefulness of tumour marker levels in pleural effusions of malignant and benign origin. Clin Chim Acta 2000;300:43-55.

16. Li CS, Cheng BC, Ge W, et al. Clinical value of CYFRA21-1, NSE, CA15-3, CA19-9 and CA125 assay in the elderly patients with pleural effusions. Int J Clin Pract 2007;61:444-8.

17. Kimura $Y$, Fujieda S, Takabayashi T, et al. Conventional tumor markers are prognostic indicators in patients with head and neck squamous cell carcinoma. Cancer Lett 2000;155:163-8.

18. Barak V, Meirovitz A, Leibovici V, et al. The Diagnostic and Prognostic Value of Tumor Markers (CEA, SCC, CYFRA 21-1, TPS) in Head and Neck Cancer Patients. Anticancer Res 2015;35:5519-24.

19. Ji M, Li XD, Shi HB, et al. Clinical significance of serum ferritin in elderly patients with primary lung carcinoma. Tumour Biol 2014;35:10195-9.

20. Kalousová M, Krechler T, Jachymova M, et al. Ferritin as an independent mortality predictor in patients with pancreas cancer. Results of a pilot study. Tumour Biol 2012;33:1695-700.

Cite this article as: Tang YF, Li W, Yuan JP, Huang YB, Yan HL, Liu L, Li XP, Wang M. Diagnostic values of serum tumor markers CA72-4, SCCAg, CYFRA21-1, NSE, AFU, CA125, CA19-9, CEA and FER in nasopharyngeal carcinoma. Transl Cancer Res 2018;7(6):1406-1412. doi: 10.21037/tcr.2018.10.24 